\title{
AN OPTIMAL SOLUTION FOR IMAGE EDGE DETECTION PROBLEM USING SIMPLIFIED GABOR WAVELET
}

\author{
C.Sujatha ${ }^{1}$ and Dr. D. Selvathi ${ }^{2}$ \\ ${ }^{1}$ Asso.Prof., Department of ECE, Sethu Institute of Technology,, \\ Kariapatti, Tamil Nadu, India \\ sssujathac@yahoo.co.in \\ ${ }^{2}$ Professor, Department of ECE, Mepco Schlenk Engineering College, \\ Sivakasi,Tamil Nadu, India. \\ selvathi_deyahoo.com
}

\begin{abstract}
Edge detection plays a vital role in computer vision and image processing. Edge of the image is one of the most significant features which are mainly used for image analyzing process. An efficient algorithm for extracting the edge features of images using simplified version of Gabor Wavelet is proposed in this paper. Conventional Gabor Wavelet is widely used for edge detection applications. Due do the high computational complexity of conventional Gabor Wavelet, this may not be used for real time application. Simplified Gabor wavelet based approach is highly effective at detecting both the location and orientation of edges. The results proved that the performance of proposed Simplified version of Gabor wavelet is superior to conventional Gabor Wavelet, other edge detection algorithm and other wavelet based approach. The performance of the proposed method is proved with the help of FOM, PSNR and Average run time.
\end{abstract}

\section{KEYWORDS}

Gabor wavelet, Simplified Gabor wavelet, edge detection, Haar wavelet, Debauchies, Symlet, Biorthogonal, Figure of Merit, Peak Signal to Noise ratio.

\section{INTRODUCTION}

In image processing and computer vision, edge detection is a process which attempts to capture the significant properties of objects in the image [1]. An edge is defined by a discontinuity in gray level values of an image. Edge detection is a fundamental tool used in most image processing applications to obtain information from the frames as a precursor step to feature extraction and object segmentation. Edges are predominant features in images and their analysis and detection are an essential goal in computer vision and image processing. Edge detection is one of the key stages of image processing and objects recognition [2]. An edge is defined by a discontinuity in gray level values. In other words, an edge is the boundary between an object and the background. The shape of edges in images depends on many parameters: geometrical and optical properties of the object, the illumination conditions, and the noise level in the images [3].

Research in automatic edge detection has been active because of this topic's wide range of applications in image processing, such as automated inspection of machine assemblies, diagnosis

DOI : 10.5121/ijcseit.2012.2307 
in medical imaging, and topographical recognition [4]. Edge detection is a very difficult task. When viewing an image, humans can easily determine the boundaries within that image without needing to do so consciously. However, no single edge-detection algorithm, at present, has been devised which will successfully determine every different type of edges [5]. Many edge detection algorithms have been proposed and implemented. These algorithms differ from each other in many aspects such as computational cost, performance and hardware implementation feasibility.

Simplified version of Gabor Wavelets (SGW) is proposed in this work, whose features can be computed efficiently and can achieve better performance for edge detection. Proposed SGWs can replace the GWs for real time applications.

\subsection{State of the art}

An edge is in general a border which separates the adjacent zones of image having distinct brightness. The development of an edge detector is often based on a specific characteristic of the image [6]. An important property of the edge detection method is its ability to extract the accurate edge line with good orientation, and much literature on edge detection has been published in the past three decades. It is encountered in the areas of feature selection and feature extraction in Computer Vision [7]. An edge detector accepts a digital image as input and produces an edge map as output. The edge maps of some detectors include explicit information about the position and strength of the edges and their orientation.

Most of the edge detection methods can be classified into five categories based on the behavioural study of edges of the images

- Gradient Based Image Edge Detection

- Zero Crossing Based Image Edge Detection

- Gaussian Based Image Edge Detection

- Laplacian of Gaussian Based Image Edge Detection

- Transform Based Image Edge Detection

\subsubsection{Gradient Based Image Edge Detection}

Gradient based edge detectors contain classical operators and uses first directional derivative of gray level gradient of an image in the spatial domain. This edge detectors have no smoothing filter, and they are only based on a discrete differential operator [8]. The earliest popular works in this category include the algorithms developed by Sobel (1970), Prewitt (1970), Kirsch (1971), Robinson (1977), and Frei-Chen (1977) [9]. They compute an estimation of gradient for the pixels, and look for local maxima to localize step edges [10]. Typically, they are simple in computation and capable to detect the edges and their orientation, but due to lack of smoothing stage, they are very sensitive to noise and inaccurate. Compass operators are enhanced version of the gradient operators. More computations are required in the compass operators in order to detect more edges and produce better results [2].

A statistical noise estimation kernel based on Rayleigh distribution function is discussed in [11]. This kernel is used to implement the breast lesion boundary detection for ultrasound images. The detection sequence for this proposed method includes such as histogram equalization, nonlinear diffusion, convolution of the breast image with the developed kernel, median filtration, segmentation, labelling and morphological operations. 
International Journal of Computer Science, Engineering and Information Technology (IJCSEIT), Vol.2, No.3, June 2012

\subsubsection{Zero Crossing Based Image Edge Detection}

Zero crossing edge detectors uses second derivatives of the image. These detectors identified that the zero crossing points in the second derivatives are considered as edge pixels of the image [12]. Zero crossing detectors include Laplacian operator and second directional derivative. The advantages of these operators are capable to detect the edges with their orientation and these operators having fixed characteristics in all direction. The main drawbacks of these operators are reresponsing to some of the existing edges and very sensitive to noise [13]. General zero crossing based discontinuity detectors in two dimensions such as Marr-Hildreth (1980), residual, difference of Gaussians, and other detectors are discussed in [14]. Mathematical justification for this class of detectors is presented in this paper. An optimal detector that maximizes the signal to noise ratio is derived. Preliminary experimental results for real images are reported in [14].

\subsubsection{Gaussian Based Image Edge Detection}

Gaussian based edge detection is symmetric along the edges and noises can be reduced by smoothing operation. There are many contributions in the last two decades that present edge detectors using either directly the Gaussian function or filters with high similarity to the Gaussian function and its derivatives. This leads us to believe that the optimum linear filter for the detection of step edges should not differ too much from the derivative of the Gaussian and therefore, the smoothing filter should be based on the Gaussian function. This is not surprising since the Gaussian has been emerging as a very important function in several areas of image analysis and processing and specially in multiresolution analysis. The significant operators in this classification are Canny and Shen-Castan. Canny edge detector convolves the image with the derivative of Gaussian to detect the edges [1]. The Canny detector first smoothes the image to eliminate noise then finds the image gradient to highlight regions with high spatial derivatives. Then the detector suppresses the non maximum pixel along the edge direction. The gradient array is further reduced by Hysteresis Thresholding technique [1].

Noise-robust edge detector is proposed in [15], which combines a small-scaled isotropic Gaussian kernel and large-scaled anisotropic Gaussian kernels to obtain edge maps of images. Its main advantage is that noise reduction is attained while maintaining high edge resolution. Anisotropic directional derivatives are derived by using anisotropic Gaussian kernals to capture the locally directional variation of an image. The performance of this method is proved using the aggregate test receiver-operating-characteristic (ROC) curves and the Pratt's Figure of Merit (FOM) [15].

\subsubsection{Laplacian of Gaussian (LoG) Based Image Edge Detection Operator}

Another well known operator based on the occurrence of zero crossings known as Laplacian of Gaussian operator [16]. Since not all zero crossings correspond to edges, some false edges may be introduced. Canny's operator [1] is one of the most widely used edge detection algorithms in the computer vision community because of its performance. In this algorithm, edge pixels are detected based on first derivative of that pixel. In addition, two thresholds are applied to remove false edges. The problem with this operator is that these two thresholds are not easily determined and low threshold produces false edges, but a high threshold misses important edges.

The Laplacian of Gaussian (LoG) operator for edge detection has been proposed by Marr and Hildreth [17]. Laplacian is a scalar estimation of the second derivative and it is orientation independent filter. It can't detect edges at corners, curves, and at locations where image intensity function varies in a nonlinear manner along an edge. The advantages of the Gaussian filter make it unique and so important in edge detection. It is proven that when an image is smoothed by a Gaussian filter, the existing zero crossings (i.e. detected edges) disappear as 
International Journal of Computer Science, Engineering and Information Technology (IJCSEIT), Vol.2, No.3, June 2012

moving from fine to coarse scale, but new ones are never created. This unique property makes it possible to track zero crossings (i.e. edges) over a range of scales, and also gives the ability to recover them at sufficiently small scales [17].

\subsubsection{Transform Based Edge Detection Methods}

Analyzing an image at different scales increases the accuracy and reliability of edge detection. Progressing between scales also simplifies the discrimination of edges versus textures. Because of having this ability, wavelet transform is an advantageous option for image edge detection in different applications. Wavelet based multi resolution expansions provide compact representations of images with regions of low contrast separated by high contrast edges [18]. Additionally, the use of wavelets provides a way to estimate contrast value for edges on a space varying basis in a local or global manner as needed.

In the context of image processing, wavelet transform (WT) is defined as the sum over the entire of rows and columns of the image intensity function multiplied by scaled and shifted versions of the mother wavelet function. The scale represents the function by compressing or stretching it, and denotes its features in frequency domain, while the shift corresponds to the translation of the wavelet function in the spatial domain. There is a correspondence between scale and frequency, a low scale shows the rapidly changing details of the intensity function with a high frequency, and a high scale illustrates slowly changing coarse features with a low frequency. Therefore, wavelet transform acts as a mathematical microscope, in which one can monitor different parts of an image by just adjusting focus on scale.

Brannock and Weeks [3] have proposed an edge-detection method based on the discrete wavelet transform (DWT), which combines DWT with other methods to achieve an optimal solution to edge-detection algorithm. Y.P.Guan [19] has proposed a multiscale wavelet edge detection algorithm for lip segmentation. In noiseless images with high contrast, Canny's edge detection has proven to be very successfu .But that algorithm is not efficient for noisy image. For noisy images, $\mathrm{Lu}$ and Zhang has proposed algorithm to detect diagonal edge information, based on the wavelet transform with shifted coefficients [3].

An edge detection algorithm based on the multi-direction shear transform is proposed in [20]. By using wavelet transform edges can be detected more accurately than gradient based methods but that edges are discontinuous. Multi-direction shear transform based edge detection is used to solve this problem [20]. Edge detection method in DCT domain for compressed images is presented in [21]. In this algorithm, gradient patterns of the image are generated using DCT. The quantized coefficients are used to calculate the edge maps, which avoids the procedure of inverse quantization. It is shown that the proposed scheme leads to not only good edges but also computation speedup [21].

Multiscale edge detection is proposed in [22]. Firstly, the nonsubsampled contourlet transform is used to decompose the original image into low frequency approximation image and the high frequency subbands. Then, because of nonsubsampled contourlet transform shift-invariance, the original image corresponding gradient magnitude is redefined in every scale. Finally, different scales gradient is synthesized into the image edge. The experimental results of edge detection for several test images are provided to demonstrate the approach [22]. An overview of various edge and line oriented approaches to contour detection in the last two decades is discussed in [23]. Proposed taxonomy of the main contour detectors presented in this literature. In this survey paper the main features and most representative articles for each class of contour is tabulated. A Robust iris recognition system using log Gabor wavelet and Laplacian of Gaussian filter is presented in [24]. This paper presented a straightforward approach for segmenting the iris patterns. This 
method determined an automated global threshold and the pupil centre. A novel method for iris recognition which utilizes both the intensity gradient and statistical analysis for feature extraction is presented in [24].

The contour detection and extraction of knee joint in CT images is implemented in [25]. The contour is detected using the first order differential operators, such as Roberts Cross operator and Sobel operator and second order differential operator, such as Laplacian and Canny operators. The chain code method is used for the contour extraction. Image reconstruction using local competition Gabor wavelets transformation is described in [26]. This algorithm is based on linear Gabor wavelets followed by a nonlinear iterative algorithm consisting of local competitions between coefficients which aim is reducing the amount of information to be encoded. The resulting transform is aliasing free and provided the exact reconstruction by the linear inverse transform.

Two novel corner detection methods for grey level images based on log Gabor wavelet transform is presented in [27]. The input image is decomposed at multiscale and along multi orientations. In the first algorithm, the corners are detected by the magnitude along the direction that is orthogonal to the gradient orientation. In this detector, corners are detected and localized accurately. The second proposed method is based on log Gabor wavelets and second moment matrix. The input image is decomposed by the log Gabor wavelets at multiscale along multi orientations. Then the components at different scales and orientations are projected onto the axis and formulated into the second moment matrix. Finally, the smaller eigenvalue of the second moment matrix is used to detect corner points.

The remaining part of this paper is organised as follows. Section 2 describes conventional Gabor Wavelet and edge detection using Gabor wavelets. Section 3 describes about proposed methodology of this work. Section 4 discusses about the obtained results and discussions. Section 5 discusses the conclusion of the proposed work

\section{CONVENTIONAL GABOR WAVELET}

Gabor Wavelets (GWs) [28] are commonly used for extracting local features for various applications such as object detection, recognition and tracking. The human visual system can be viewed as composed of a filter bank. The responses of the respective filters can be modeled by Gabor functions of different frequencies and orientations. The Gabor features have been found to be particularly appropriate for texture representation and discrimination and have been successfully applied to texture segmentation, face recognition, handwritten numerals recognition, and fingerprint recognition. In the spatial domain, a 2D Gabor filter is a Gaussian kernel function modulated by a sinusoidal plane wave as follows:

$$
G(x, y)=\exp \left[-\frac{x^{2}+y^{2}}{2 \sigma^{2}}\right] \exp [\mathrm{j} \omega(x \cos \theta+y \sin \theta)]
$$

Where $\sigma$ is the standard deviation of the Gaussian function in the $\mathrm{x}$ - and $\mathrm{y}$-directions and $\omega$ denotes the spatial frequency. Family of Gabor kernels can be obtained from eqn.(1) by selecting different center frequencies and orientations. These kernels are used to extract features from an image. 


\subsection{Edge detection using Conventional Gabor wavelet}

Gabor wavelets can effectively abstract local and discrimination features. In textural analysis and image segmentation, GW features have achieved outstanding results, while in machine vision, they found to be effective in object detection, recognition and tracking. The most useful application of the Gabor Wavelets is for edge detection [28].For given an input image $\mathrm{I}(\mathrm{x}, \mathrm{y})$, the Gabor Wavelet features are extracted by convolving $\mathrm{I}(\mathrm{x}, \mathrm{y})$ with $\mathrm{G}(\mathrm{x}, \mathrm{y})$ as in equation (2).

$$
\Phi(x, y)=G(x, y) \otimes I(x, y)
$$

Where $\otimes$ denotes the 2-D convolution operation [19]. The Gabor wavelets (GWs) respond strongly to edge if the edge direction is perpendicular to the wave vector $(\omega \cos \theta, \omega \sin \theta)$. When hitting an edge, the real and imaginary parts of $\Phi(\mathrm{x}, \mathrm{y})$ oscillate with the characteristic frequency in- stead of providing a smooth peak.

\section{PROPOSED WORK}

The computation required for Gabor Wavelet based feature extraction is very intensive. This in turn creates a bottleneck problem for real time processing. Hence, an efficient method for extracting Gabor features is needed for many practical applications.

\subsection{Simplified Gabor Wavelets}

Wei Jiang.et.al.[28]have proposed that the imaginary part of a Gabor filter is an efficient and robust means for edge detection. The imaginary part of a GW is as in equation (3):

$$
S(x, y)=\exp \left[-\frac{x^{2}+y^{2}}{2 \sigma^{2}}\right] \sin [\omega(x \cos \theta+y \sin \theta)]
$$

Edges can be detected by using this simplified Gabor Wavelet. Set of Simplified Gabor kernels can be obtained from eqn. (3) by selecting different center frequencies and orientations. These kernels are used to extract features from an image. This method is known as Simplified Gabor wavelet.

\subsection{Shape of an SGW}

The equation for 1-D Gabor Wavelet is shown in equation (4).

$$
s(x)=\frac{1}{2 \prod \sigma^{2}} \exp \left[-\frac{x^{2}}{2 \sigma^{2}}\right] \sin (\omega x)
$$

The values of imaginary part of 1-D GW are continuing one. Its values are quantized to a certain number of levels. The same number of quantization levels is used for the positive and the negative values of the Gabor function because it is antisymmetrical [4]. For 2-D cases, the imaginary part of a 2-D GW, with the gray-level intensities representing the magnitudes of the Gabor function. 


\subsection{Determination of Quantization Levels}

The determination of the quantization levels for an SGW is the same as that in [29]. One of the quantization levels of the SGW is set to zero. As the imaginary part of a Gabor function is antisymmetrical, the number of quantization levels for the positive and negative values are equal and are denoted as $n_{1}$. Then, the total number of quantization levels is $2 n_{1}+1$. Suppose that the largest magnitude of the $\mathrm{GW}$ is $\mathrm{A}$, the corresponding quantization levels for positive levels and negative levels are as in equation (5)

$$
q_{+}(k)=\frac{A}{2 n_{1}+1} \cdot 2 k \cdot q_{-}(k)=-\frac{A}{2 n_{1}+1} \cdot 2 k
$$

where $\mathrm{k}=1 \ldots \mathrm{n}_{1}$. These SGWs are then convolved with an image to extract the SGW features at different center frequencies and orientations to form a simplified Gabor jet.

\subsection{Determination of the Parameters}

The values of important parameters for the GWs or SGWs are determined for edge detection, which are the values of $\omega, \sigma$, and $\theta$. Edges of an image can be detected in different directions, by setting different values for $\theta$ [30]. Computational can be reduced by setting four values for $\theta$. Hence, the number of orientations used in this proposed work is four,i.e., $\theta_{k}=k \pi / 4$ for $k=0,1,2,3$. As edges are much localized feature of an image, the value of $\omega$ should be small when compared to that for face recognition [4]. So, in this proposed work edges can be detected efficiently by setting $\omega=0.3 \pi$ and $0.5 \pi$.

\subsection{Efficient Edge Detection Using SGWs}

Edge detection can be done efficiently by using SGWs of two different scales $(\omega)$ and four different orientations $(\theta)$. The convolution of an SGW of scale $\omega$ and orientation $\theta$ with the image $\mathrm{I}(\mathrm{x}, \mathrm{y})$ generates the $\mathrm{SGW}$ features and is denoted as $\phi_{\omega, \theta}^{\prime}\left(x_{c}, y_{c}\right)$. The resulting $\mathrm{SGW}$

feature $\phi^{\prime \prime}{ }_{\omega, \theta}\left(x_{c}, y_{c}\right)$ at a pixel position $\left(x_{c}, y_{c}\right)$ is equal to the absolute maximum of the eight $\phi_{\omega, \theta}^{\prime}\left(x_{c}, y_{c}\right)$, i.e.,

$$
\phi^{\prime \prime}{ }_{\omega, \theta}\left(x_{c}, y_{c}\right)=\max \left\{\phi_{\omega i, \theta j}^{\prime}\left(x_{c}, y_{c}\right), i=0,1 \text { and } j=0, \ldots, 3\right\}
$$

where $\omega_{0}=0.3 \pi, \omega_{1}=0.5 \pi$, and $\theta_{\mathrm{j}}=\mathrm{j} \pi / 4$, for $\mathrm{j}=0, \ldots, 3$. The SGW feature $\phi_{\omega, \theta}^{\prime}\left(x_{c}, y_{c}\right)$ is computed by convolving the image I(x,y) with the SGW whose patterns are dependent on the scale $\omega$ and the orientation $\theta$. As edges are much localized in an image, so the window size of the patterns is either $3 \times 3$ or $5 \times 5$. The SGWs are formed using two levels of quantization for the positive and the negative magnitudes of the GWs. These two quantization levels are denoted as q1 and $\mathrm{q} 2$ with $\mathrm{q} 2>\mathrm{q} 1$ for positive magnitudes and the corresponding quantization levels for the negative magnitude are $-\mathrm{q} 1$ and $-\mathrm{q} 2$, respectively. Two different scales and four different orientations are adopted for this proposed work. The required computation for a $\phi_{\omega, \theta}^{\prime}\left(x_{c}, y_{c}\right)$ is not more than 2 multiplications and 22 additions. Hence the computational cost is very lower than conventional Gabor Wavelet [30]. 
International Journal of Computer Science, Engineering and Information Technology (IJCSEIT), Vol.2, No.3, June 2012

\section{RESULT AND DISCUSSION}

The performance of the proposed SGW based approach with different scales and different orientations are evaluated. Then relative performance with the use of the GW features and the SGW features were compared. Performance of the Canny, Sobel, Robert, Prewitts and conventional Gabor wavelet operators were compared with SGW based Edge Detection Algorithm. The performance of the proposed SGW is also compared with other wavelet transform based approach such as Haar wavelet, Biorthogonal wavelet, Symlet, and Debauchies Then this proposed work performance is also proved with noisy image also.

\subsection{Performance Analysis of SGWs with Different Quantization Levels for Edge Detection}

The effect of the number of quantization levels on edge detection using Simplified Gabor Wavelet can be evaluated using three, five and seven quantization levels. In this analysis, the coins images and cameraman images are used as shown in Fig.1 and Fig 2. The edge detection results based on SGW with $\omega=0.3 \pi$ and three different quantization levels for coin image are shown in Fig.1(a) 1(d).And for the same image, result for $\omega=0.5 \pi$ and three different quantization levels are shown in Fig.1(e) - 1(g). Fig.2(a)-2(g) shows the results for cameraman image for $\omega=0.3 \pi$ and $\omega=0.5 \pi$ with three different quantization levels.

From Fig.1 and Fig. 2, the performance of edge detection using SGWs of five and seven quantization levels are better than three quantization levels, while the performances of five and seven quantization levels are very similar. More computation can be required for higher number of quantization levels. Hence, five quantization levels are chosen in this proposed work. 


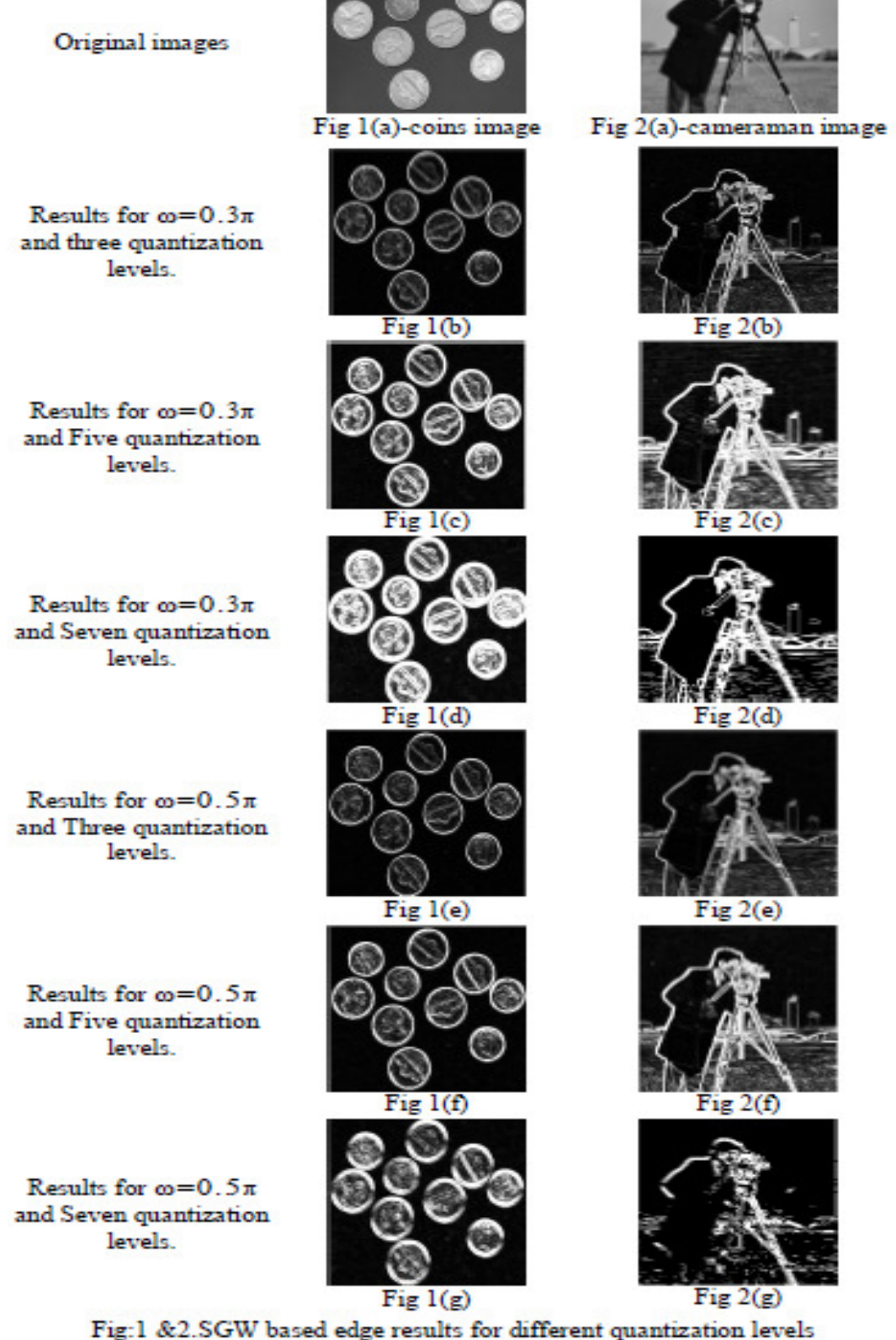

\subsection{Performance Analysis of SGWs with Different Scales for Edge Detection}

The most promising performance in terms of accuracy and computation can be achieved by using SGWs with five quantization levels, which is proved in Sec 4.1. This section evaluates the effect of SGWs with five quantization levels and with different scales $\omega=0.125 \pi, \omega=0.3 \pi, \omega=0.5 \pi$ and $\omega=0.65 \pi$. The edges of the cameraman image and coins image based on SGW features of the four different scales are shown in Fig 3 and Fig 4. 
International Journal of Computer Science, Engineering and Information Technology (IJCSEIT), Vol.2, No.3, June 2012

The edge detection results based on SGW with five quantization levels and four different scale $\omega=0.125 \pi, \omega=0.3 \pi, \omega=0.5 \pi$ and $\omega=0.65 \pi$. of cameraman image are shown in Fig 3(a) $-3(\mathrm{e})$. And the Fig. 4(a)-4(e) shows the results of coin image for $\omega=0.125 \pi, \omega=0.3 \pi, \omega=0.5 \pi$ and $\omega=0.65 \pi$ with five different quantization levels. From this comparison, two scales $\omega=0.3 \pi, \omega=0.5 \pi$ can be identified for better performance.

\subsection{Comparing the performance of SGWs with other Edge Detection Algorithms}

The performance of SGWs based edge detection algorithm are compared with some conventional edge detection algorithms, such as Canny, Sobel, Prewitt, Robert and conventional Gabor Wavelet methods. In order to get best performance, the SGWs with two scales $\omega=0.3 \pi, \omega=0.5 \pi$, four orientation $\theta=\{0, \pi / 4, \pi / 2,3 \pi / 4\}$, and five quantization levels are used for this comparision.Fig.5 shows the results of various edge detection algorithm for cameraman image, satellite image, Angiogram brain image and pears image. This result comparison proved that proposed Simplified Gabor Wavelet is optimal than other conventional method and it is an efficient algorithm for all type of images.

\subsection{Comparing the performance of SGWs with other Wavelet based Edge Detection Algorithms}

The performance of SGWs based edge detection algorithm are compared with other wavelet based methods such as Haar, Biorthogonal,Symlet, and Debauchies wavelet methods. In order to get best performance, the SGWs with two scales $\omega=0.3 \pi, \omega=0.5 \pi$, four orientation $\theta=\{0, \pi / 4$, $\pi / 2,3 \pi / 4\}$, and five quantization levels are used for this comparision.Fig.6 shows the results of various edge detection algorithm for cameraman image, satellite image, Angiogram brain image and pears image. This result comparison proved that proposed Simplified Gabor Wavelet is an efficient than all other wavelet based methods.

\subsection{Performance comparison of SGWs with other Edge Detection Algorithms for noisy image}

The performance of the proposed work is evaluated for noisy image also. For this comparison noisy images are obtained by adding white Gaussian of zero mean and 0.01 variance. For this comparison also, the SGWs with two scales $\omega=0.3 \pi, \omega=0.5 \pi$, four orientation $\theta=\{0, \pi / 4, \pi / 2$, $3 \pi / 4\}$, and five quantization levels are used in order to get best performance. The results comparison of proposed Simplified Gabor Wavelet method for noisy images with other edge detection operator is given in Fig.7. This result proved that the Simplified Gabor Wavelet method is better than Haar wavelet, Biorthogonal and Debauchies wavelet. Thus the proposed work provides efficient edge detection for all type of images even in the presence of noise. 


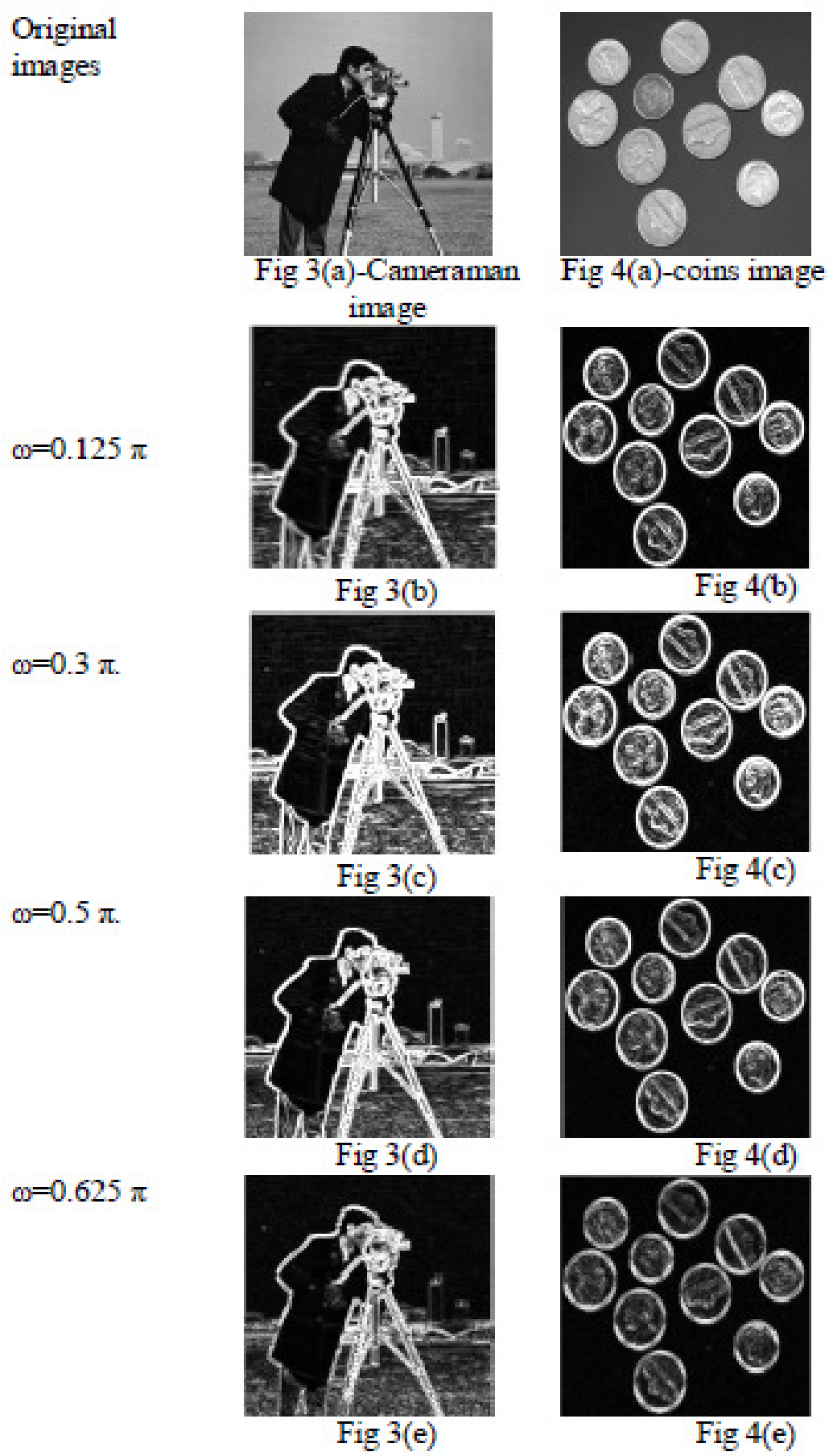

Fig: $3 \& 4$ SGW based edge detection results for different values of $\omega$. 


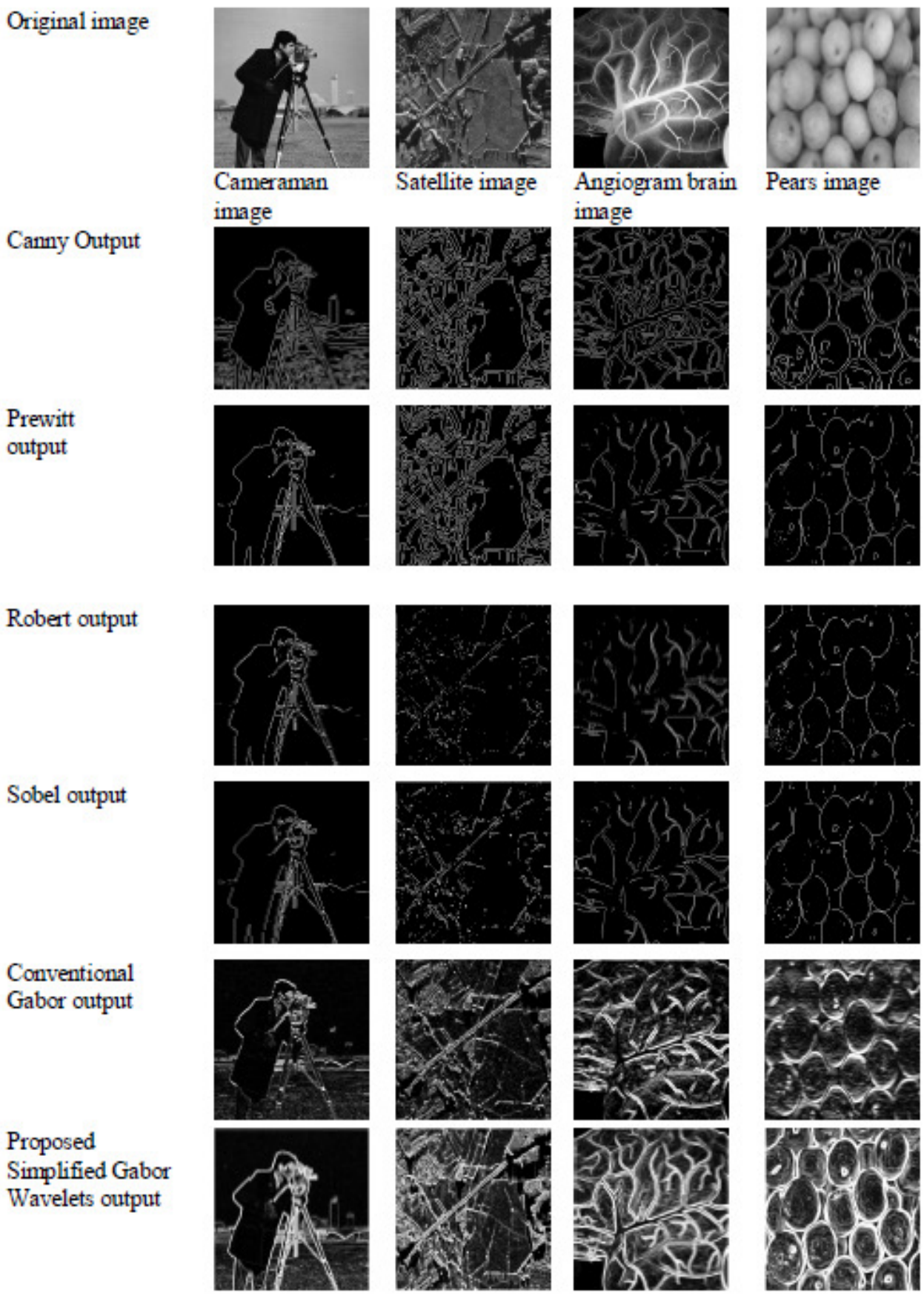

Fig.5.Comparison of Simplified Gabor Wavelet results with different algorithms 


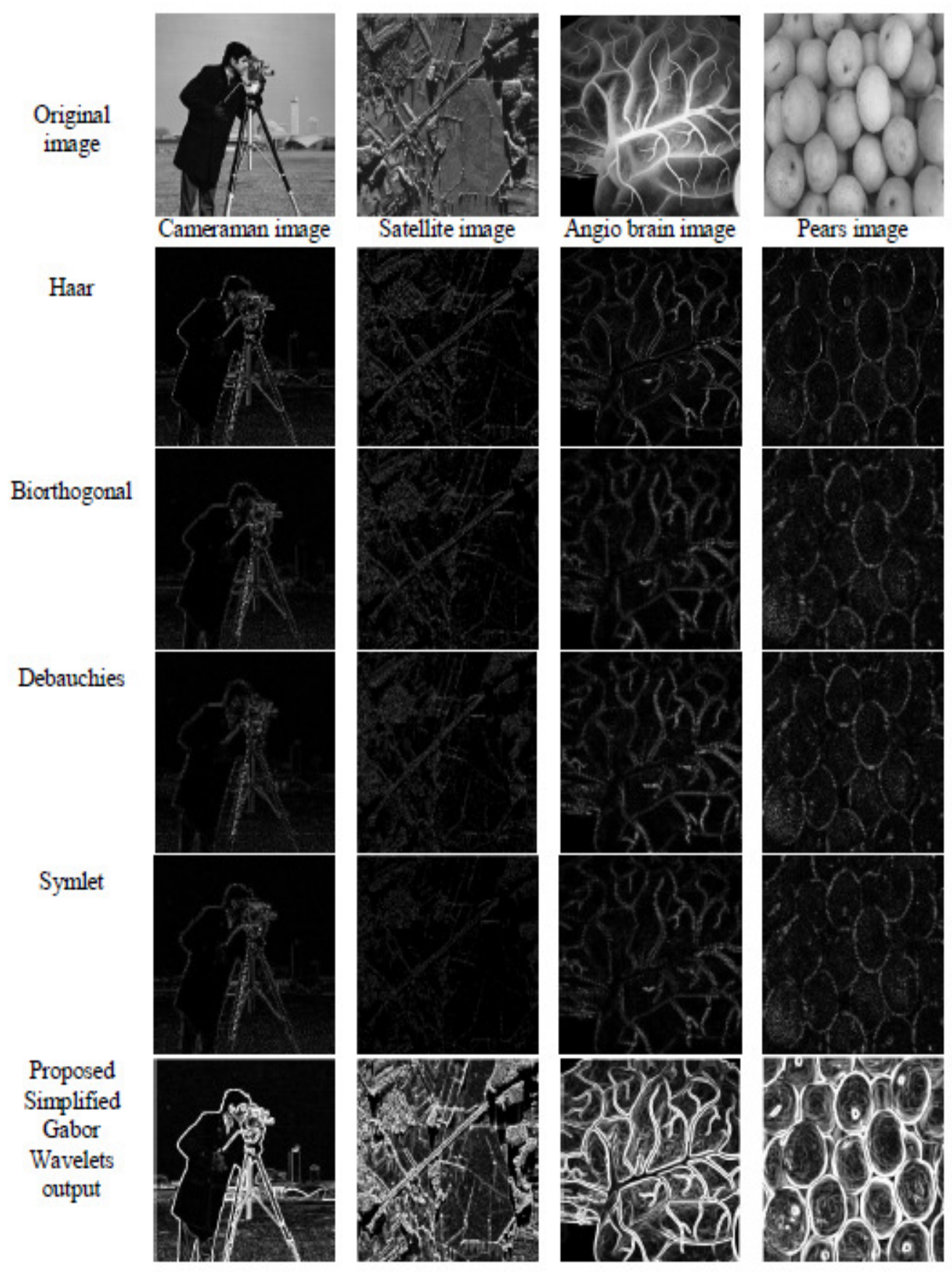

Fig.6 .Comparison of Simplified Gabor Wavelet results with different wavelet algorithms 

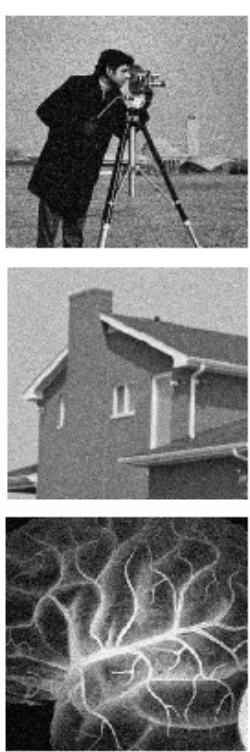

Original image
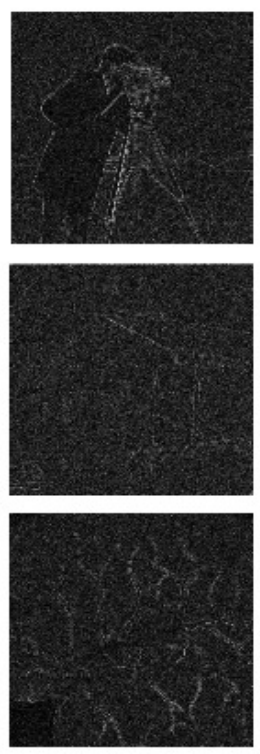

Haar
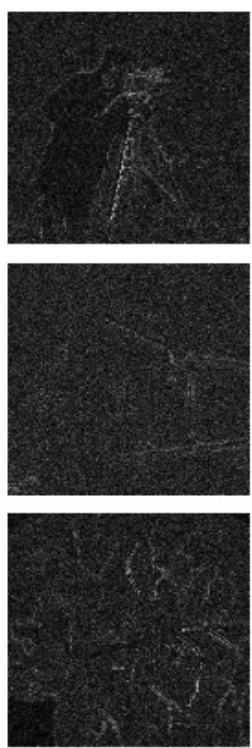

Debauchies
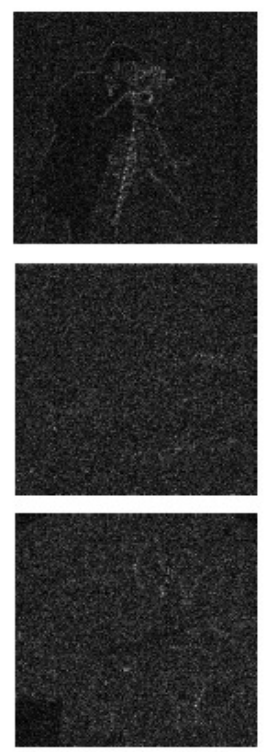

Biorthogonal
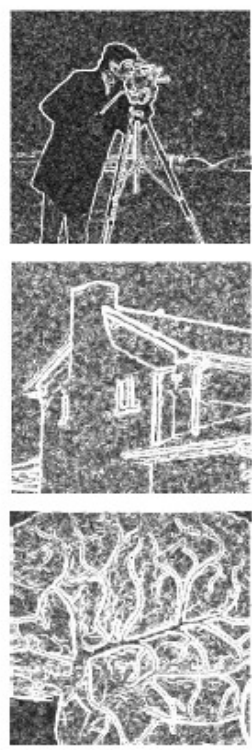

Proposed SGW

Fig.7. Comparison of Proposed Simplified Gabor Wavelet results with other edge detection methods for noisy images

\subsection{Comparison of Quantitative Analysis for SGWs with other edge detection algorithms}

The performance comparison was discussed in last section. The Quantitative measures for all the edge detection algorithm is described in this section. For quantitative analysis of the proposed method performance measures for cameraman image such as average run time and PSNR are calculated and are tabulated in Table 1 and Table 2 for different edge detection algorithms respectively. The average runtime required by SGWs is compared with other conventional methods. This comparison is tabulated in Table1. The run time required by SGW based edge detection algorithm is smaller than that required by conventional Gabor Wavelet and Canny edge detection methods. The average run time is similar to that of Prewitt and Roberts's method, but compared with their performances the proposed Simplified Gabor Wavelet is superior to all other methods.

Table 1. Average Run Time of Various methods

\begin{tabular}{|c|c|c|}
\hline S.No & Algorithm & $\begin{array}{c}\text { Average } \\
\text { Run Time }\end{array}$ \\
\hline 1 & Canny & $76.5 \mathrm{~ms}$ \\
\hline 2 & Prewitt & $23.4 \mathrm{~ms}$ \\
\hline 3 & Roberts & $21.8 \mathrm{~ms}$ \\
\hline 4 & Sobel & $78 \mathrm{~ms}$ \\
\hline 5 & Gabor Wavelet & $47 \mathrm{~ms}$ \\
\hline 6 & $\begin{array}{c}\text { Simplified Gabor } \\
\text { Wavelet }\end{array}$ & $31 \mathrm{~ms}$ \\
\hline
\end{tabular}


Peak Signal to Noise Ratio is measured between edge images of original image and noisy image. In this PSNR measure, Gaussian noise is added to the original image and that is considered as noisy image. PSNR values for different algorithms are compared with proposed SGW based edge detection. Those measures are tabulated in Table.2.

Table 2. PSNR of Various methods

\begin{tabular}{|c|c|c|}
\hline S.No & Algorithm & $\begin{array}{c}\text { PSNR in } \\
\mathrm{db}\end{array}$ \\
\hline 1 & Canny & 8.45 \\
\hline 2 & Prewitt & 17.45 \\
\hline 3 & Roberts & 16.61 \\
\hline 4 & Sobel & 17.77 \\
\hline 5 & Gabor Wavelet & 25.23 \\
\hline 6 & $\begin{array}{c}\text { Simplified Gabor } \\
\text { Wavelet }\end{array}$ & 33.41 \\
\hline
\end{tabular}

From this quantitative analysis the proposed Simplified Gabor Wavelet based edge detection provides better performance than other conventional methods. Fast run time of proposed work realize that it is most suited for real time application.

\subsection{FOM Comparison of SGW with other edge detection algorithms}

The edge detector performance must be proved by both qualitative and quantitative measures. Various quantitative measures are used to measure the performance of an edge detector. From the above PSNR and Average run time comparison, proposed work performance was proved. The other most recognized measure is Pratt's Figure of Merit (FOM) [16]. The FOM is based on the combination of three factors: non detection of true edges, detection of false edges and edge delocalization error, and it is defined as in equation (7)

$$
F=\frac{1}{\max \left(N_{e}, N_{d}\right)} \sum_{k=1}^{N_{d}} \frac{1}{1+\alpha d(k)^{2}}
$$

Where $N_{e}$ is the number of actual edge points, $N_{d}$ is the number of detected edge points, $d(k)$ is the distance from the $\mathrm{k}^{\text {th }}$ actual edge point to the detected edge point and $\alpha$ is a scaling constant set to 0.1 . By considering the canny output image as actual edge map, the Figure of Merit (FOM) is calculated for SGW based edge results for various images. And for the same images FOM values for Sobel, Robert, Haar, Debauchies wavelet based edge results are measured. And its values are tabulated in Table 3. These comparisons proved that the FOM for Simplified Gabor Wavelet results are higher than all other methods.

Table 3. Figure of Merit for Various Images

\begin{tabular}{|c|c|c|c|c|c|}
\hline Original Image & Sobel & Robert & Haar & Debauchies & SGW \\
\hline Cameraman & 0.5034 & 0.4731 & 0.1369 & 0.1261 & 0.6671 \\
\hline Building & 0.4686 & 0.5385 & 0.1942 & 0.1501 & 0.5341 \\
\hline Brain & 0.5969 & 0.5658 & 0.1271 & 0.1418 & 0.8108 \\
\hline Pears & 0.4132 & 0.5695 & 0.1667 & 0.1169 & 0.5011 \\
\hline
\end{tabular}




\section{CONCLUSION}

In this paper, an efficient algorithm for image edge detection using simplified version of Gabor Wavelets is proposed. Proposed work is based on the conventional Gabor wavelet, but it can detect more edge pixels than the conventional one. The various experiments prove that proposed algorithm clearly outperforms than other conventional and wavelet based edge detection methods. The performance of proposed algorithm is also proved for noisy images also. The proposed algorithm very effectively used for biomedical images also. The results prove that the edge features of angiographic brain images using proposed Simplified Gabor Wavelet are better than other conventional methods. The quantitative measures show that proposed SGW based edge detection is fast and better PSNR than other conventional methods. The SGW method has higher FOM than all other methods. The performance comparisons and quantitative analysis proves that the proposed Simplified Gabor Wavelet based edge detection is very much suited for real time applications.

\section{REFERENCES}

[1] J.Canny, (1986) “A computational approach to edge detection,” IEEE Trans. Pattern Anal. Mach. Intell., Vol.8, No. 6, pp. 679-698.

[2] Fahad M.Alzahrani \& Tom Chen, (1997), "A Real Time Edge Detector: Algorithm and VLSI Architecture", Journal of Real-Time Imaging, Vol. 3,pp. 363-378.

[3] E. Brannock \& M. Weeks, (2008) "A synopsis of recent work in edge detection using the DWT", Proc. IEEE Southeastcon, pp. 515-520.

[4] Wei Jiang, Kin-Man Lam \& Ting-Zhi Shen, (2009) "Efficient Edge Detection Using Simplified Gabor Wavelets," IEEE Transactions on Systems, Man and Cybernetics- Part B: Cybernetics,pp.1-11.

[5] Jian-Jun Hao, Qiang Jiang, Jian-Wei Wei, Lin Mi, (2010) "Research of Edge Detection Based on Gabor Wavelet", IEEE International Conference on Measuring Technology and Mechatronics Automation, Vol. 2, pp.1083-1086.

[6] B.Bouda,Masmoudi \& D.Aboutajdine, (2007) "A New Model for Edge Detection in Digital Images", GVIP Special issue on Edge detection, Vol.7, pp.25-30.

[7] Rosenfel, A. (1986) "Computer vision: a source of models for biological visual process", IEEE Trans. on Biomedical Engineering, Vol. 36, Iss. 1, pp.93 - 96.

[8] Gonzalez.R \& Woods R.E (1992), Digital Image Processing, Addision- Wesley Publishing Co.Inc.

[9] Mohsen Sharifi, Mahmoud Fathy, Maryam Tayefeh Mahmoudi (2002) "A Classified and Comparative Study of Edge Detection Algorithms", IEEE Int. conf. on Information technology: Coding and computing, pp.117 - 120.

[10] Sobel, I. (1978) "Neighborhood coding of binary images fast contour following and general array binary processing", Elsevier Computer Graphics and Image Processing, Vol. 8, Iss.1, pp.127-135.

[11] Alwaleed Abdelrahman, Omer Hamid (2011) "Lesion boundary detection in ultrasound breast images", IEEE Conf. on Biomedical Engineering, pp.320-323.

[12] Albovik (2000) Handbook of Image and video processing, Academic Press.

[13] Raman Maini, Himanshu Aggarwal, Dr.(2010), "Study and Comparison of Various Image Edge Detection Techniques', Int. Jour. of Image Processing”, Vol. 3, Iss.1, pp.1-12.

[14] Lee,D., Wasilkowski, G.W., Mehrotra, R. (1993), "A new zero-crossing-based discontinuity detector", IEEE Trans. on Image Processing, Vol.2, Iss.1, pp.265 - 268.

[15] Peng-Lang Shui, Wei-ChuanZhang (2012), "Noise-robust edge detector combining isotropic and anisotropic Gaussian kernels", Elsevier Pattern Recognition, vol. 45, pp.806-820.

[16] W. K. Pratt (1978), Digital Image Processing, New York: Wiley-Interscience.

[17] Marr, D., Hildreth, E.C. (1980), "Theory of edge detection", Proc. of the Royal Society of London. Series B, Biological Sciences, Vol.207, Iss.1167, pp.187-217.

[18] Stephane Mallat (1991) "Zero-Crossings of a Wavelet Transform”, IEEE Trans. on Information Theory, Vol.37, Iss.44, pp. 1019- 1033.

[19] Y.P.Guan (2008) "Automatic extraction of lips based on multi-scale wavelet edge detection," Computer Vision, Vol.2, No.1, pp.23-33.

[20] Pengfei Xu, Qiguang Miao, Cheng Shi, Junying Zhang, Weisheng Li, (2012) “An edge detection algorithm based on the multi-direction shear transform”, Elsevier Journal of Visual Communication Image Representation, vol. 23, pp. 827-833.

[21] Zhenxing Qiana, Wenwen Wang, Tong Qiao, (2012) “An Edge Detection Method in DCT Domain”, Elsevier Procedia Engineering, Vol. 29, pp. $344-348$. 
[22] Chen Zhigang ,Cui Yueli \& Chen Aihua,(2012) “An Multi-scale Edge Detection Approach”, Elsevier Physics Procedia, Vol. 25, pp.1616 - 1620.

[23] Giuseppe Papari, Nicolai Petkov, (2011) "Edge and line oriented contour detection: State of the art", Elsevier Image and Vision Computing, Vol. 29, pp.79-103.

[24] Bhawna Chouhan, Shailja Shukla, (2011) "Comparative Analysis of Robust Iris Recognition System Using Log Gabor Wavelet and Laplacian Of Gaussian Filter", Int. Jour. of Comp. Science and Communication, Vol.2, Iss.1, pp.239-242.

[25] Xushu Zhang, Yuan Guo, Peng Du, (2011) “The Contour Detection and Extraction for Medical Images of Knee Joint", IEEE Int. Conf. on Bioinformatics and Biomedical Engineering, pp. 1 - 4.

[26] Sylvain Fischer, Gabriel Cristobal, Rafael Redondo, (2006) "Sparse Over complete Gabor Wavelet Representation Based on Local Competitions" IEEE Trans. on Image Processing, Vol.15, Iss.2, $\quad$ pp. 265 272.

[27] Xinting Gao, Farook Sattar, Ronda Venkateswarlu, (2007) "Multiscale Corner Detection of Gray Level Images Based on Log-Gabor Wavelet Transform", IEEE Trans. on Circuits and Systems for Video Technology, Vol.1, Iss.7, pp. $1253-1256$

[28] Wei Jiang, Ting-Zhi Shen, Jian Zhang, Yu Hu, Xin-Yi Wang, (2008) "Gabor Wavelets for Image Processing", IEEE International Colloquium on Computing, Communication, Control, and Management, Vol.1.pp.110_114.

[29] R. Mehrotra, K. Namuduri, \& N. Ranganathan, (1992) "Gabor filter-based edge detection", Pattern Recognition, vol.25, Iss.12, pp.1479-1494.

[30] W. P. Choi, S. H. Tse, K. W. Wong \& K. M. Lam, (2008) "Simplified Gabor wavelets for human face recognition”, Pattern Recognition, Vol. 41, No.3,pp. 1186-1199.

\section{Authors}

Dr.D.SELVATHI received her B.E degree in Electronics and Communication Engineering from Mepco Schlenk Engineering College, Sivakasi, Tamilnadu, India in 1988 and M.S. degree in Electronics and Control from Birla Institute of Technology and Science, Pilani, India in 1994. She completed her Ph.D degree in the area of Medical Image Processing at Manonmanium Sundranar University, Tirunelveli, Tamilnadu, India in 2008. Presently, she is working as Professor, Department of Electronics and Communication Engineering, Mepco Schlenk Engineering College, Sivakasi, Tamilnadu, India.She published more than 80 papers including 15 papers in international journals and 2 papers in national journals. She received two best paper awards and YOUNG SCIENTIST FELLOWSHIP AWARD from Tamilnadu State Council for Science and Technology in the year 2000-2001. She is the principal investigator for two R\&D projects received from AICTE, New Delhi. She organized and attended many conferences and seminars. At present she is guiding $9 \mathrm{Ph} . \mathrm{D}$ scholars. Her research interests are Image Processing for Medical applications, Soft Computing, Kernel methods and Pattern Recognition. This includes Medical images classification and pattern recognition using Support Vector Machines and Neural Networks and Medical images analysis.

Mrs.C.SUJATHA received B.E degree in Electronics and Communication Engineering from P.S.N.A College of Engineering and Technology, Dindigul, Tamil Nadu, India in 1997 and M.E degree in Applied Electronics from P.S.N.A College of Engineering and Technology, Dindigul, Tamil Nadu, India in 2004. She has more than 10 years of teaching experience. Presently she is working as Associate Professor, Department of Electronics and Communication Engineering, Sethu Institute of Technology, Kariapatti, Tamil Nadu, India. She is doing research in the area of Image Processing based on

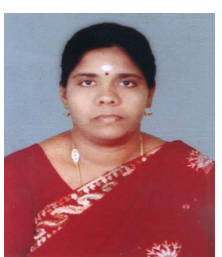
FPGA under the guidance of Dr.D.Selvathi, Prof. /ECE Dept., Mepco Schlenk Engineering College, Sivakasi,. Tamilnadu, India. 